\title{
La información periodística de portada en España: evolución histórica de fuentes, agendas y encuadres (1980-2010)
}

\author{
Pablo LóPEZ-RABADÁN \\ Universitat Jaume I \\ rabadan@uji.es \\ Andreu CASERo-RIPOLLÉS \\ Universitat Jaume I \\ casero@uji.es
}

\begin{abstract}
Resumen:
Dentro de la cobertura informativa, la portada de la prensa de referencia representa un objeto de estudio muy relevante. A partir su análisis longitudinal, el objetivo de este trabajo es examinar la evolución de tres cuestiones periodísticas centrales: gestión de fuentes, composición de la agenda informativa, y tipo de encuadre dominante. Tras analizar una muestra de 800 noticias, los resultados indican un aumento progresivo en la cantidad y diversidad de fuentes; una agenda bastante estática y tradicional; y un patrón de encuadre caracterizado por el conflicto y el equilibrio entre lo temático y lo episódico.
\end{abstract}

Palabras clave: Información periodística; Prensa de referencia; Evolución histórica; Fuentes informativas; Agenda mediática; Encuadre interpretativo.

\section{The cover news reporting in Spain: historical evolution of sources, agenda and frames (1980-2010)}

\begin{abstract}
:
In news coverage, the cover of press reference represents a very important subject of study. From a longitudinal analysis, the aim of this article is to examine the evolution of three crucial journalistic issues: sources management, media agenda composition, and dominant framing type. After analyzing a sample of 800 news, results indicate a progressive increase in the number and diversity of sources; an agenda rather static and traditional; and a framing pattern characterized by conflict and balance between the thematic and episodic.
\end{abstract}

Key Words: Journalistic information; Press reference; Historical evolution; News sources; Media Agenda; Interpretive frame.

\section{Referencia normalizada:}

López Rabadán, P. y Casero Ripollés, A. (2014): La información periodística de portada en España: evolución histórica de fuentes, agendas y encuadres (1980-2010). Historia y Comunicación Social. Vol. 19. Núm. Especial Enero. Págs. 457-473.

Sumario: 1. Introducción. 1.1. Tres componentes decisivos de la información periodística: fuentes, agendas y encuadres. 2. Metodología. 3. Resultados. 3.1. Las fuentes informativas. 3.2. La construcción de la agenda mediática. 3.3. Encuadre. 4. Conclusiones. 5. Referencias bibliográficas. 6. Nota. 


\section{Introducción}

La información periodística juega un papel clave en nuestra sociedad. Se configura como un recurso esencial para el ejercicio de la ciudadanía democrática puesto que posibilita la participación política, la formación de la opinión pública y el acceso al debate social. La importancia de estos aspectos, vinculados al ejercicio de la influencia social en el terreno simbólico, justifica el estudio de su contenido.

El objetivo de este trabajo es examinar la configuración y evolución de tres cuestiones centrales en el proceso de producción de la información periodística que determinan su contenido y, por lo tanto, su potencial influencia: la gestión de fuentes, la composición de la agenda informativa y el tipo de encuadre interpretativo dominante. En este sentido, se han estudiado, a partir del análisis de contenido, los siguientes indicadores: número de fuentes, tipo de identificación y tipo de actores-fuente (1); temas de la agenda informativa y homogeneidad temática (2); y clasificación del encuadre según las principales tipologías de referencia (3).

1.1 Tres componentes clave de la información periodística: fuentes, agendas y encuadres

Las fuentes informativas ocupan una posición central en el periodismo (Manning, 2001). Los periodistas las necesitan para que les aporten informaciones relevantes y dignas de confianza (Berkowitz, 2010). La gestión de fuentes asume un papel esencial en el proceso de producción de noticias y de construcción de la realidad (Casero-Ripollés, 2008; Grossi, 2007; Carlson, 2011). Las fuentes se sitúan en una posición intermedia entre el acontecimiento y el periodista, cosa que les permite desplegar una actividad de mediación entre ambas esferas que resulta estratégica y condicionante (Borrat, 2006; Diezhandino, 2009).

La investigación científica ha puesto de manifiesto diversos rasgos sobre el papel de las fuentes en la información periodística. En primer lugar, diversos estudios demuestran el protagonismo de las fuentes oficiales (Tuchman, 1978; Gans, 1980; Dimitrova \& Strömbäck, 2009). La información periodística se convierte, así, en un mecanismo al servicio de las elites políticas y contribuye decisivamente al mantenimiento del statu quo social y político. En segundo lugar, otros trabajos detectan un déficit estructural en el número de fuentes en el periodismo actual (Mayoral, 2005; De Pablos, 2006; García-Santamaría, 2010) y un aumento de la presencia de fuentes anónimas (Franklin \& Carlson, 2011). Ambas cuestiones repercuten directamente en importantes problemas de calidad, transparencia y credibilidad profesional. En tercer término, varios estudios revelan la profesionalización de las fuentes, especialmente las políticas, a partir de la acción de los gabinetes comunicación. Éstos influyen en la producción de noticias, mediante actividades de relaciones públicas, dificultando el acceso directo a fuentes primarias y poniéndose al servicio de poder político y económico (Davis, 2002; Lewis et al., 2008). 
Un segundo aspecto esencial en la producción de la información periodística tiene que ver con la composición de la agenda informativa o del temario periodístico (De Fontcuberta, 2011). Su importancia está determinada por el hecho que la cobertura periodística influye en las prioridades y preocupaciones de los ciudadanos. Algo que se produce, especialmente, en los momentos excepcionales y críticos (Casero-Ripollés, 2004). Esta circunstancia ha sido puesta de manifiesto empíricamente por la teoría de agenda setting (McCombs \& Shaw 1972). Esta perspectiva describe el proceso mediante el cuál los medios periodísticos presentan ciertos temas de manera frecuente y prominente con el resultado que amplios sectores del público perciben esas cuestiones como más importantes que otras, activando, así, el proceso de formación de la opinión pública (McCombs, 2004).

Y por último, un tercer elemento determinante dentro del proceso de producción periodística es la construcción de encuadres interpretativos dominantes. Desde hace varias décadas, se viene produciendo una aplicación progresiva en el campo comunicativo del concepto de encuadre (frame) o marco de referencia acuñado por Erving Goffman (1974/2006). En la actualidad es una destacada línea de investigación que aborda muy diferentes objetos de estudio vinculados a la producción, contenido y efectos sociales e individuales de los medios de comunicación (Kamhawi \& Weaver, 2003; Bryant \& Miron, 2004; Weaver, 2007). A pesar de que se ha caracterizado por una notable dispersión teórica y metodológica, actualmente se detecta un sólido avance de esta línea de investigación a nivel internacional y un arranque paulatino en España (Vicente-Mariño \& López-Rabadán, 2009; López-Rabadán, 2010).

Es posible subrayar tres convenciones bastante generalizadas a la hora de plantear el análisis del encuadre mediático (López-Rabadán \& Vicente-Mariño, 2013). La primera es localizar el encuadre entorno a dos procedimientos profesionales básicos, la selección y la organización discursiva (Reese, Grant \& Gandy, 2001; Tewksbury y Scheufele, 2007). Ante la gran resistencia de los medios a desvelar directamente sus estrategias informativas, la segunda convención es la necesidad de realizar aproximaciones empíricas indirectas y a medio plazo, tomando como objeto de análisis su proyección sobre un periodístico completo (Matthes \& Kohring, 2008), sobre el que tratar de identificar funciones de encuadre (Entman, 1993), palabras clave o tipologías concretas (entre otros, Gamson \& Modigliani, 1989; Iyengar, 1991; Semetko \& Valkenburg, 2000). Y la tercera, es la importancia de desarrollar diseños metodológicos que combinen el análisis de contenido cuantitativo y la introducción de categorías de carácter cualitativo (Matthes \& Kohring, 2008).

En suma, las fuentes informativas, la configuración de la agenda informativa y el enfoque o framing de las noticias son elementos esenciales en la producción de la información periodística. Sin embargo, además son aspectos clave en la construcción periodística de la realidad ya que de su configuración depende la influencia mediática sobre la sociedad. Por ello, resulta fundamental el estudio de cómo se han configurado estos contenidos de la información periodística en España desde una perspectiva histórica que analice el período que va desde 1980 a 2010. 


\section{Metodología}

La técnica de investigación aplicada es el análisis de contenido cuantitativo. El modelo de análisis se centra en tres grandes aspectos: las fuentes informativas, la agenda temática y el framing o enfoque de las noticias. Para su estudio se adopta una perspectiva de análisis longitudinal para identificar con precisión tendencias estructurales a largo plazo, a partir del análisis de una amplia muestra de la prensa española de los últimos 30 años (1980-2010). La muestra temporal se ha delimitado en dos pasos: seleccionando los años iniciales de cada década (1980, 1990, 2000, 2010); y dentro de cada año, seleccionando 100 días del primer semestre para aplicar el análisis de contenido cuantitativo. La muestra está integrada por 800 noticias.

En cuanto a los medios, se han seleccionado dos ejemplos relevantes dentro de la prensa de referencia española: los diarios El País y $A B C$. Ambos tiene una posición muy destacada en el panorama mediático nacional durante todo el periodo analizado, y los dos presentan una línea editorial claramente diferenciada: progresista o centro-izquierda en el primer caso, y conservadora o centro-derecha en el segundo. Y por ultimo, la muestra se termina de delimitar definiendo la unidad de registro sobre la que aplicar la parrilla de análisis de contenido. En este caso, por motivos operativos y de relevancia profesional se ha optado por analizar únicamente la noticia principal dentro de la portada. Ésta es un espacio mediático preferente dónde se representan con la máxima claridad los estándares profesionales y los criterios de selección y jerarquización de un determinado medio de comunicación (Davara-Torrego y otros, 2004).

\section{Resultados}

\subsection{Las fuentes informativas}

\subsubsection{Número de fuentes por noticia}

El porcentaje de noticias en las que se detecta una ausencia ( «Sin fuentes») o escasez de fuentes («Una fuente»), aunque relativamente preocupantes, quedan limitadas al 20\% del total (gráfico 1). Por su parte, aquellas que se ajustan al estándar mínimo de calidad periodística («Dos fuentes» o «Tres fuentes») alcanzan una importancia central dentro del conjunto con el $34,7 \%$ del total. También adquieren un peso destacado el resto de categorías que ya indican una riqueza notable en el empleo de fuentes en la información: «Cuatro fuentes» $(16,9 \%)$, «Cinco fuentes» $(13,4 \%)$ y «Más de cinco fuentes» $(14,3 \%)$. 
Gráfico 1. Número total de fuentes informativas por noticia.( $\mathrm{N}=800$ portadas).

\section{NÚMERO DE FUENTES (total 1980-2010)}

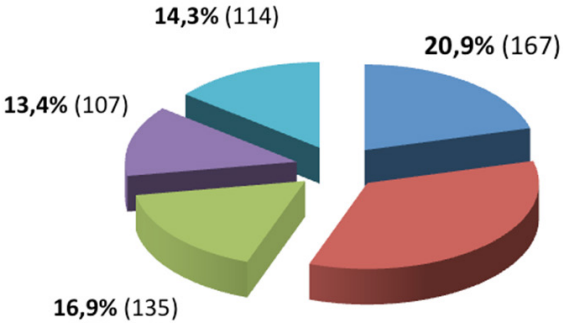

$34,7 \%(277)$

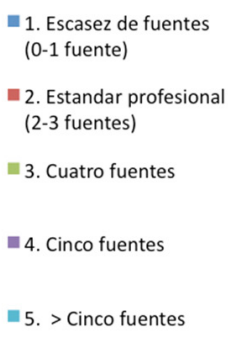

Fuente: Elaboración propia

Es posible identificar un aumento progresivo en el número de fuentes por noticia entre 1980 y 2010, que acabada consolidándose en el tiempo (gráfico 2). En este sentido, cabe señalar que entre 1980 y 2010, las categorías que indican déficit de fuentes («Sin fuentes» $\mathrm{y}$ «Una fuente») pasan de tener un peso relativo del $23 \%$ entre ambas (47 informaciones), a reducir su presencia hasta el 13\% (26 informaciones), con la práctica desaparición de las noticias de portada sin fuentes (sólo tres casos en 2010). Por otra parte, las categorías que representan el estándar profesional mínimo («Dos fuentes» $\mathrm{y}$ «Tres fuentes»), mantienen constante un porcentaje mayoritario que oscila entre el $35 \%$ y el $38 \%$ durante todo el ciclo. El cambio más relevante es el experimentado por los tres grupos clasificatorios con mayor riqueza y diversidad de fuentes (a partir de cuatro fuentes por información). Éstos pasan a convertirse, considerados conjuntamente, en la categoría más numerosa en 2010 llegando al 51,5\% del total de las noticias (103 noticias).

\subsubsection{Identificación de fuentes}

Los resultados globales del periodo analizado (1980-2010) indican un cumplimento bastante generalizado de la identificación de las fuentes dentro de la información en la prensa española (gráfico 3). Estos datos conectan con criterios de excelencia periodística como la transparencia, la credibilidad y la influencia. En este sentido, las dos categorías principales («Correctamente identificadas»y «Parcialmente identificadas») representan casi el $89 \%$ dentro del total de 2760 fuentes localizadas en la muestra, siendo claramente mayoritario el grupo que ofrece una identificación completa y correcta de las fuentes $(68,6 \%)$. De esta forma, el problema de las fuentes veladas queda reducido a nivel menor, pero siempre mejorable, del 11,1\% de los casos. 
Gráfico 2. Resumen de la evolución (1980-2010) del número total de fuentes por noticia ( $\mathrm{N}=800$ portadas).

\section{NÚMERO DE FUENTES (Evolución 1980-2010)}

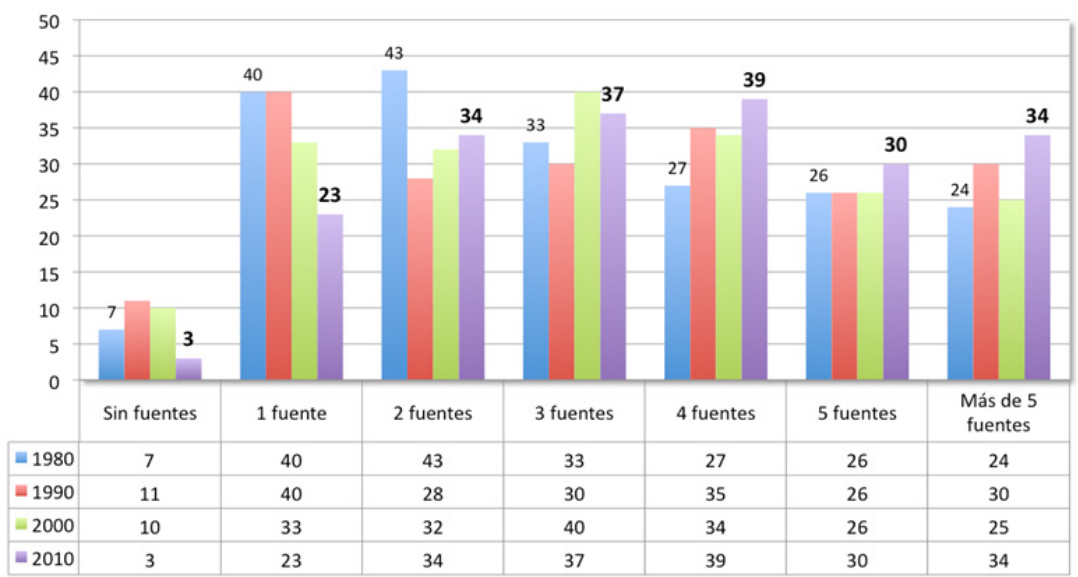

Fuente: Elaboración propia

Gráfico 3. Tipo de identificación de las fuentes en las noticias ( $\mathrm{N}=2760$ fuentes).

\section{TIPO IDENTIFICACIÓN FUENTES (Total 1980-2010)}

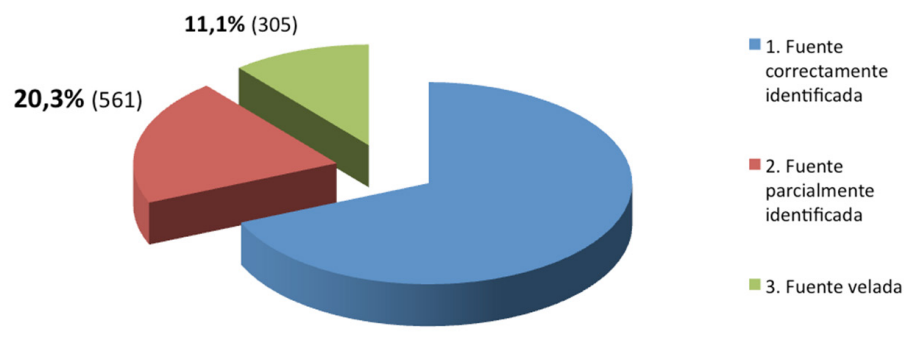

$68,6 \%(1896)$

Fuente: Elaboración propia

La evolución de la atribución de fuentes entre 1980 y 2010 plantea dos aspectos importantes (gráfico 4). En primer lugar, conviene remarcar que la identificación correcta de fuentes se mantiene en 2010 en un nivel muy similar o ligeramente superior al de 30 años atrás, quedando en el 68,3\% de los casos (514 fuentes). No obstante, como contraparte, se detecta un preocupante aumento de la fuentes veladas en el periodo temporal estudiado. En este sentido, el número de fuentes anónimas 
ha crecido desde el 5,8\% (41 fuentes) en 1980, al 13\% (98 fuentes) en 2010. Estos resultados evidencian un problema profesional en la prensa española que oscurece de forma significativa su proceso de producción informativa, dañando la transparencia del mismo.

Gráfico 4. Resumen de la evolución (1980-2010) de la identificación de fuentes (N=2760 fuentes).

TIPO IDENTIFICACIÓN FUENTES (Evolución 1980-2010)

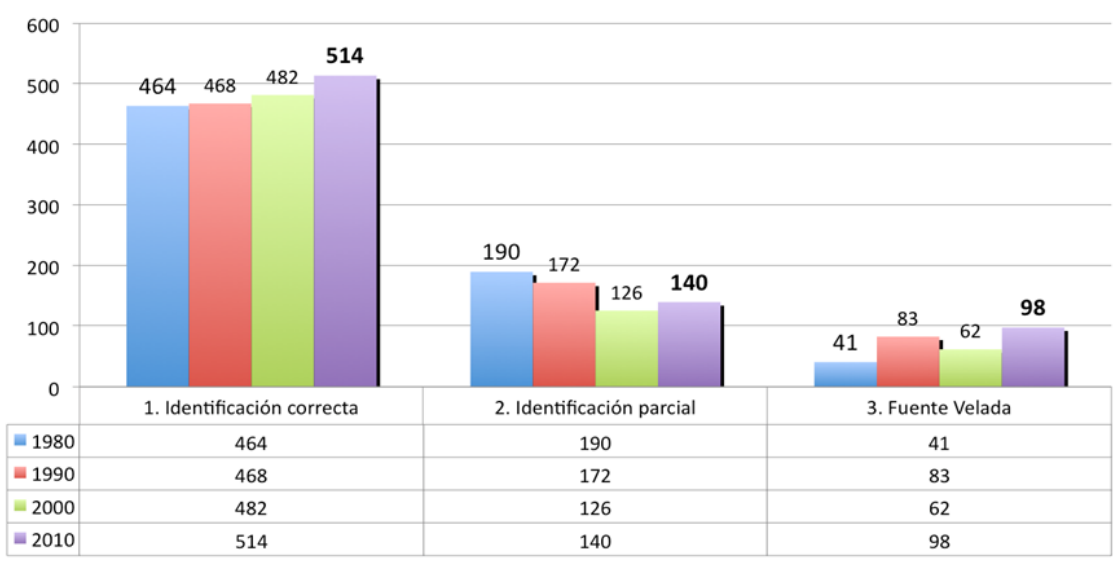

Fuente: Elaboración propia

\subsubsection{Tipología de las fuentes}

Los resultados indican un peso mayoritario de las fuentes personales, que se configuran con la principal vía informativa para los periodistas en el 83,2\% de los casos (2297 fuentes). Frente a ellas, las documentales sólo alcanzan el 16,8\% (463 fuentes). Estos datos confirman la existencia de unas rutinas profesionales basadas en el trato directo con las fuentes y un relativo déficit en lo que se refiere a la consulta de documentación especializada.

En lo referente a la clasificación de fuentes, los resultados globales y la evolución a lo largo del periodo analizado (1980-2010) corroboran el dominio de las fuentes oficiales, vinculadas a todo tipo de instituciones públicas. Esta circunstancia limita el contraste de puntos de vista en la información y, en consecuencia, reduce la pluralidad del debate social.

Los resultados totales del periodo analizado permiten identificar tres niveles diferentes de importancia (gráfico 5). En el primero, con gran diferencia respecto del resto, sobresalen las «institucionales», que representan más de la mitad del total, concretamente un 51,6\%. En este grupo se integran todo tipo de administraciones 
públicas, siendo el Ejecutivo estatal (17,8\%) y las internacionales $(13,8 \%)$ las más referenciadas. Por sus evidentes conexiones, también se incluyen a las fuentes «políticas», partidos y grupos parlamentarios, que representan el 12,1\%. En un segundo nivel se sitúan las fuentes de la «sociedad civil». Éstas suponen el 10,3\% del total. En esta categoría se engloban, fundamentalmente, ciudadanos individuales, sindicatos, asociaciones y ONG. Finalmente, en un tercer escalón se localizan varios grupos sociales con una importancia reducida en cuanto a su uso como fuentes informativas. Son los casos del «sistema mediático», vinculado los medios de comunicación tradicionales $(7,3 \%$ del total), las fuentes «económicas», principalmente las empresas ( $6 \%$ del total), y las «alternativas», dónde se engloban los expertos científicos $(5,8 \%$ del total).

Gráfico 5. Tipología de fuentes informativas ( $N=2760$ fuentes)

TIPOLOGÍA FUENTES INFORMATIVAS (Total 1980-2010)

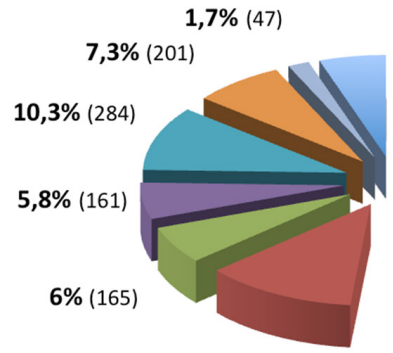

$12,1 \%(333)$

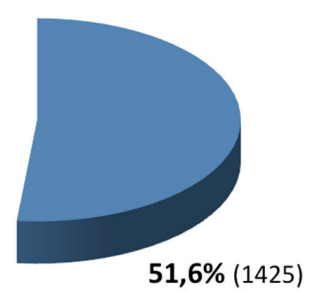

$51,6 \%(1425)$
1. Institucionales

2. Políticas

=3. Económicas

4. Alternativas

5. Sociedad civil

6. Sistema MMCC

=7. Culturales

$=8$. Otras

Fuente: Elaboración propia

Sobre la evolución a lo largo del tiempo se detectan varias tendencias significativas (gráfico 6). En primer lugar, se reafirma el peso mayoritario y constante de las fuentes «institucionales» como principal referente informativo en la prensa española. Aunque registran, incluso, un crecimiento en las cifras totales, de 346 fuentes (1980) a 394 (2010), el porcentaje total se mantiene prácticamente igual, ya que sólo varía 3 décimas a lo largo del ciclo analizado. En segundo lugar, llama la atención el comportamiento irregular de las fuentes «políticas». Esta circunstancia provoca que vean reducido sensiblemente su peso relativo dentro de la estructura de fuentes, pasando del 14,2\% en 1980 (86 fuentes) al 10,6\% en 2010 (73 fuentes).

Finalmente, en tercer lugar, es interesante subrayar el crecimiento relativo, en distinta medida, del resto de grupos sociales. En este sentido, las fuentes clasificadas como «sociedad civil» sufren un ligero incremento, pasando del 9,1\% (55 fuentes) a casi un 12\% (81 fuentes). Con ello, logran superar a las fuentes «políticas» en 2010. Aunque con cifras totales modestas y de manera irregular, también registran aumentos las fuentes «alternativas»y «económicas». Por ultimo, es importante recalcar la 
reducción en la presencia de las fuentes «culturales» que prácticamente desaparecen de la estructura de fuentes de la prensa española en 2010.

Gráfico 6. Resumen de la evolución (1980-2010) de la tipología de fuentes ( $\mathrm{N}=2760$ fuentes).

\section{TIPOLOGÍA FUENTES INFORMATIVAS (Evolución 1980-2010)}

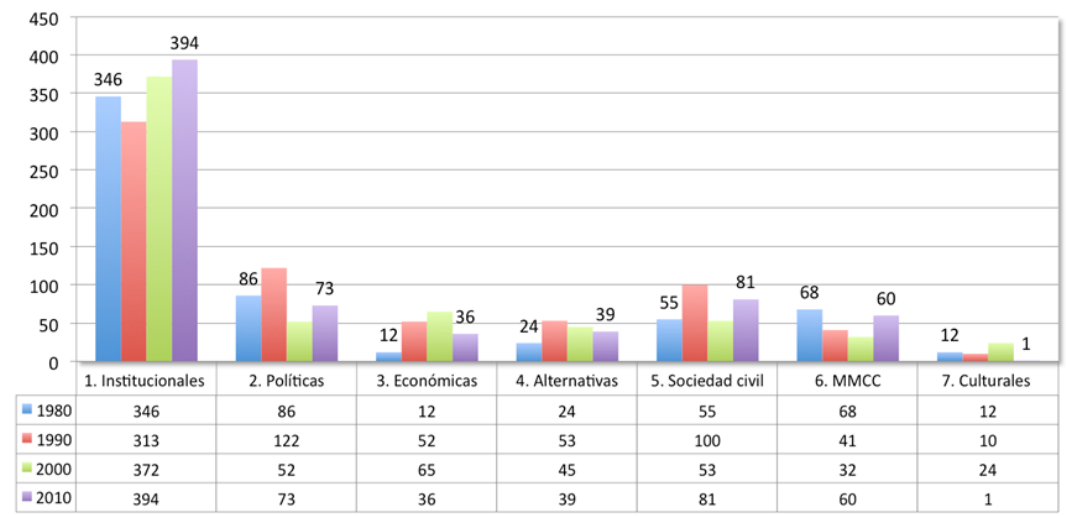

Fuente: Elaboración propia

3.2 La construcción de la agenda mediática

\subsubsection{Agenda mediática}

Respecto a la composición de la agenda mediática de la prensa española, los resultados globales del periodo analizado (1980-2010) permiten distinguir tres grandes grupos de temas, de mayor a menor importancia (gráfico 7).

El primero incluye los asuntos políticos, tanto el debate nacional español $(23,6 \%$ del total) y la política internacional $(18,8 \%)$, que se configuran claramente como las temáticas más importantes. A esto hay que añadir otros temas más específicos pero directamente relacionados con la actualidad política, como son el terrorismo $(8,4 \%)$, el funcionamiento de la Unión Europea $(6,1 \%)$ o los casos de corrupción política, referidos fundamentalmente a España $(4,1 \%)$.

Un segundo grupo temático destacable son las cuestiones de gestión vinculadas a la vida social y cotidiana de los ciudadanos. Se trata de la economía $(7,9 \%)$, el empleo (5\%), el sistema judicial $(3,8 \%)$ y la seguridad ciudadana $(2,4 \%)$.

Por ultimo, se detecta un tercer grupo caracterizado por la presencia de contenidos más ligeros, $\mathrm{y}$, al mismo tiempo, con una considerable carga de espectáculo y dramatismo informativo. Se trata de los temas de catástrofes y sucesos $(3,3 \%)$ y los deportivos $(2,6 \%)$. También dentro de este bloque se pueden englobar las noticias vinculadas al ámbito artístico y cultural (4,6\%). 
Gráfico 7. Composición global de la agenda temática $(\mathrm{N}=800$ portadas).

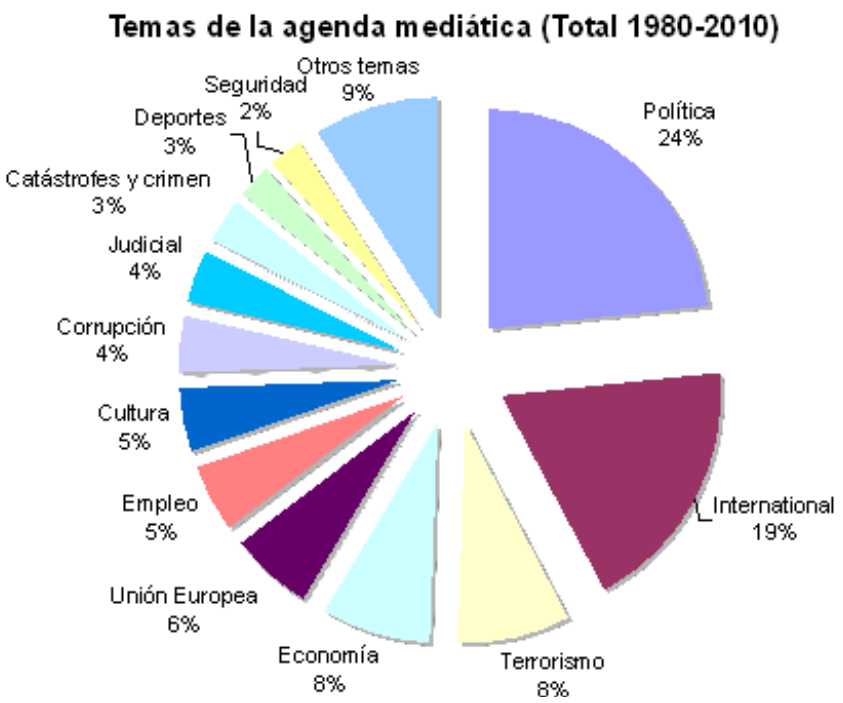

Fuente: Elaboración propia

La evolución longitudinal de los temas de portada permite identificar una potente tendencia de fragmentación temática. Partiendo de una situación inicial en 1980 dónde dos grandes temas (política nacional y actualidad internacional) configuraban cerca del $80 \%$ de la agenda de portada, a lo largo de las tres décadas han ido ganando un peso notable otros temas más específicos. Mientras las cuestiones anteriores han perdido un $60 \%$ y un $30 \%$ de su peso relativo, respectivamente, otras como la corrupción, la economía, el empleo, o el terrorismo han experimentado fuertes subidas. Casos significativos, son la estabilidad en la cobertura de la Unión Europea, que apenas sufre variaciones, y la casi desaparición de los asuntos culturales de la portada de la prensa española.

El resultado final de esta tendencia en 2010 es la consolidación de una agenda temática bastante equilibrada y plural, en la cuál 6 temas adquieren una importancia relativa muy similar. Se trata de política nacional, corrupción, terrorismo, economía, noticias internacionales y empleo. El resumen de los datos sobre esta evolución se presenta en el gráfico 8. 
Gráfico 8. Resumen evolución agenda temática ( $\mathrm{N}=800$ portadas)

\section{Temas de la agenda mediá- ca (Evolución 1980-2010)}

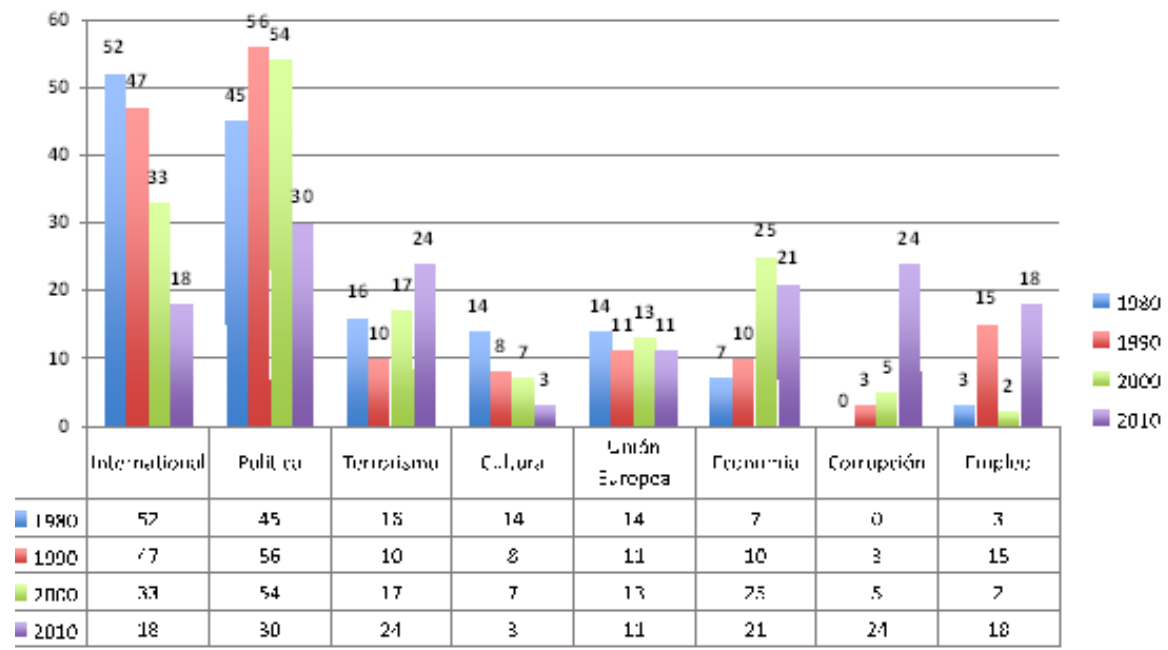

Fuente: Elaboración propia

El estudio de la dicotomía entre noticias duras (hard news) y blandas (soft news), y su evolución durante el periodo analizado, permite detectar resultados significativos. Los temas definibles como soft news alcanzan una presencia media del 19,12\% del total a lo largo de todo el periodo analizado. Además, durante los treinta años considerados no se produce un aumento de las noticias asociadas al infoentretenimiento, puesto que la diferencia entre los datos de 1980 y 2010, en cuanto a noticias blandas se refiere, es mínima, ya que se sitúa en 1,5 puntos porcentuales (tabla 1).

Tabla 1. Resumen evolución Soft News / Hard News (1980-2010)

\begin{tabular}{|c|c|c|}
\hline Año & $\begin{array}{c}\text { PROPORCIÓN } \\
\text { SOFT NEWS }\end{array}$ & $\begin{array}{c}\text { PROPORCIÓN } \\
\text { HARD NEWS }\end{array}$ \\
\hline 1980 & $20 \%(40$ portadas $)$ & $80 \%(160$ portadas $)$ \\
\hline 1990 & $15 \%(30$ portadas $)$ & $85 \%(170$ portadas $)$ \\
\hline 2000 & $15 \%(40$ portadas $)$ & $85 \%(160$ portadas $)$ \\
\hline 2010 & $21,5 \%(43$ portadas $)$ & $79 \%(157$ portadas $)$ \\
\hline Total & \multicolumn{2}{|c|}{$153 / 800=19,12 \%$} \\
\hline
\end{tabular}

Fuente: Elaboración propia 


\subsubsection{Homogeneidad de la agenda mediática}

Respecto al grado de coincidencia entre los dos diarios analizados, a la hora de seleccionar el tema principal de portada, se detecta una reducida homogeneidad temática. En el período 1980-2010, El País y ABC apenas coinciden de media en su selección un $25,5 \%$ del total de portadas analizadas. La diferencia ideológica entre sus respectivas líneas editoriales explica, en parte, un interés temático no siempre convergente. No obstante, se trata de una cifra significativamente baja, que indica con claridad la existencia de criterios noticiosos diferenciados y estrategias profesionales bastante contrapuestas. La tabla 2 recoge los datos sobre el grado de homogeneidad temática.

Tabla 2. Resumen grado de coincidencia temática de portadas $(\mathrm{N}=800)$

\begin{tabular}{|c|c|}
\hline \multicolumn{2}{|c|}{$\begin{array}{c}\text { COINCIDENCIA TEMÁTICA PORTADA } \\
\text { El País / ABC (Evolución 1980-2010) }\end{array}$} \\
\hline 1980 & $30 \%(60$ portadas $)$ \\
\hline 1990 & $17 \%(34$ portadas $)$ \\
\hline 2000 & $26 \%(58$ portadas $)$ \\
\hline 2010 & $29 \%(52$ portadas $)$ \\
\hline Total & $204 / 800=25,5 \%$ \\
\hline
\end{tabular}

Fuente: Elaboración propia

\subsection{Encuadre}

\subsubsection{Cumplimiento funciones básicas de encuadre}

Respecto a las funciones clásicas de encuadre, Robert M. Entman (1993) señala cuatro aspectos centrales que deberían tener una presencia clara en todo texto periodístico: definición del problema principal; diagnóstico de las causas que lo provocan; juicio o valoración moral al respecto; y por último, propuesta de soluciones.

En este sentido, el repaso al grado de cumplimento de estas cuatro funciones, y más desde una perspectiva histórica longitudinal de treinta años, ofrece una imagen bastante precisa de la estructura informativa de la prensa española en términos de encuadre interpretativo dominante. A continuación se presenta un resumen de los principales resultados alcanzados (ver tabla 3 ).

Sobre la primera función, "Definición del problema" (F1), detectamos un muy alto grado de cumplimiento, concretamente el 91,6\% de las noticias de portada analizadas es posible determinar con claridad la causa principal del problema analizado. Respecto a la segunda función, "Diagnóstico de las causas" (F2), detectamos un 
sensiblemente menor grado de cumplimiento. En el $66,1 \%$ de las noticias analizadas se detecta con claridad suficiente una explicación de las causas que han originado el problema objeto de la información. Sobre la tercera función, "Juicio Moral" (F3), se detecta de nuevo una muy alto grado de cumplimiento $(89,7 \%)$. De esta forma, la evaluación de las causas y sobre todo de los efectos derivados se convierte en un pilar fundamental a la hora de orientar interpretativamente el texto periodístico. Por ultimo, respecto a la cuarta función, "Propuesta de soluciones" (F4), es necesaria destacar que es la que tiene un cumplimiento claramente inferior. En sólo el 55,1\% de las noticias analizadas se identifica con claridad una oferta de remedio o el planteamiento de salidas razonadas al problema objeto de la información publicada.

Tabla 3. Resumen grado de cumplimiento funciones clásicas de encuadre $(\mathrm{N}=800)$.

\begin{tabular}{|l|c|}
\hline \multicolumn{1}{|c|}{ FUNCIÓN ENCUADRE } & GRADO CUMPLIMIENTO \\
\hline F1. Definición del problema & $91,6 \%$ (735 portadas) \\
\hline F2. Diagnóstico de las causas & $66,1 \%$ (531 portadas) \\
\hline F3. Juicio Moral & $89,7 \%$ (718) portadas \\
\hline F4. Propuesta de soluciones & $55,1 \%$ (443 portadas) \\
\hline
\end{tabular}

Fuente: Elaboración propia

\subsubsection{Uso de tipologías especificas de encuadre}

Los estudios de encuadre se han caracterizado por intentar clasificar, con mayor o menor precisión, este procedimiento profesional a través de un muy variado número de tipologías. Y dentro del diversificado conjunto, por su grado de consolidación y riqueza de análisis, se ha optado por revisar la muestra teniendo en cuenta las tres siguientes.

En primer lugar, la utilización de la tipología temática de Semetko \& Valkenburg (2000) devuelve una orientación interpretativa bastante problemática de la realidad social. En este sentido, el tipo de encuadre más importante, casi hegemónico, es el de "Conflicto" (47,42\% de los casos), claramente distanciado de los detectados en segundo ("Interés humano", 21\%) y tercer lugar ("Consecuencias económicas", $17,1 \%)$. El resto de encuadres de esta tipología tienen una presencia todavía menor en la muestra de portadas analizadas, "Moralidad" (5,8\%) y "Responsabilidad" (8,3\%).

En segundo lugar se ha revisado la muestra a partir de la tipología pionera de Shanto Iyengar (1991), muy interesante para valorar el nivel de generalización informativa. En este sentido, se comprueba una presencia bastante compensada (aunque superior del primer tipo) entre encuadres "episódicos" (55\% de los casos) que se centran en casos bastante puntuales y atribuyen una responsabilidad individual a los problemas planteados, y encuadres "temáticos" $(44,8 \%)$, que orientan el contenido informativo desde un punto de vista más amplio y atribuye las responsabilidad al conjunto de la sociedad. 
Por ultimo, también se ha revisado la muestra teniendo en cuanta la tipología de Eidels \& Lutter (2000) que presenta como criterio clasificatorio principal la base temporal empleada. De acuerdo con estos autores, el encuadre claramente hegemónico en la prensa española es el de "Diagnostico actual" (77,7\% de los casos) frente a una presencia mucho más minoritaria de aquellos que realizan una "previsión de futuro" (14\%), o adoptan una perspectiva más "motivacional" (7,6\%).

\section{Conclusiones}

Los resultados obtenidos sobre los tres componentes estratégicos de la información periodística (gestión de fuentes, planteamiento de agenda y construcción de encuadres interpretativos) permiten establecer una serie de conclusiones sobre la evolución de los contenidos de la prensa española en los últimos treinta años (1980-2010).

La consistencia de los patrones discursivos detectados delinean una estructura profesional de la gestión de fuentes en relativa transformación. Sin cambios drásticos, si se detectan varias tendencias significativas: una relativa pluralización (respecto a indicadores como el numero); un identificación ligeramente peor (debido al aumento de las fuentes veladas); y el fuerte protagonismo del poder político institucional como fuente.

Sin embargo, respecto a la construcción de la agenda mediática se dibuja estructura profesional bastante más estática y tradicional. Destaca con una fuerte y regular presencia, nunca inferior al $80 \%$, de los contenidos de temas fuertes o hard news. También sobresale significativamente el bajo grado de homogeneización temática que se sitúa en un $25,5 \%$.

Finalmente, respecto al encuadre interpretativo se detectan varios patrones discursivos bastante regulares en las últimas décadas. La cobertura tiende a orientarse a partir de dos funciones básicas, definir con claridad el problema principal, y emitir un juicio moral al respecto. Resulta bastante mejorable el grado de cumplimento de una importante función como es la propuesta de soluciones. También llama la atención el excesivo peso que tiene algunos encuadres genéricos, como el de conflicto o el de diagnostico, que tienden a reforzar una cobertura periodística con un tono excesivamente negativo y poco contextualizado.

\section{Bibliografía}

BERKOWITZ, D. A. (2010). "Reporters and their sources". En ALLAN, S. (ed.) (2010). The Routledge companion to news and journalism. London: Routledge. 
BORRAT, H. (2006). "Los periódicos, narradores en interacción". En DE FONTCUBERTA M.; BORRAT, H. (eds.) (2006). Periódicos, sistemas complejos, narradores en interacción. Buenos Aires: La Crujía.

BRYANT, J.; MIRON, D. (2004). "Theory and research in mass communication". En: Journal of Communication, $\mathrm{n}^{\mathrm{o}} 54$ (4), p. 662-704.

CARLSON, M. (2011). "Wither Anonymity? Journalism and Unnamed Sources in a Changing Media Environment". En FRANKLIN, B.; CARLSON, M. (eds.) (2011). Journalism, sources and Credibility. New Perspectives. London: Routledge. p. 37-48.

CASERO-RIPOLLÉS, A. (2004). "Los medios de comunicación ante el 11-M: la construcción de un "caso excepcional"”. En: Quaderns del CAC, $\mathrm{n}^{\circ}$ 19-20, p. $9-14$.

(2008). La construcción mediática de las crisis políticas. Madrid: Fragua.

DAVARA TORREGO, J.; et al. (2004). España en portada. Análisis de las primeras páginas de los diarios nacionales y su influencia en la sociedad. Madrid: Fragua.

DAVIS, A. (2002). Public Relations Democracy: Politics, Public Relations and the Mass Media in Britain. Manchester: Manchester University Press.

DE FONTCUBERTA, M. (2011). La noticia (nueva ed.). Barcelona: Paidós.

DE PABLOS, J. M. (2006). "Fuentes mudas en la web: periodismo transit propaganda". En: Estudios sobre el Mensaje Periodístico, no 12, p. 115-144.

DIEZHANDINO, P. (2009). Criterio noticioso. Madrid: Pearson.

DIMITROVA, D. V.; STRÖMBÄCK, J. (2009). "Look Who's Talking: Use of Sources in Newspaper Coverage in Sweden and the United States". En: Journalism Practice, $\mathrm{n}^{\circ} 3$ (1), p. 75-91.

EILDERS, C.; LÜTERS, A. (2000). "Research note: Germany at war. Competing framing estrategies in german public discourse". En: European Journal of Communication, $\mathrm{n}^{\mathrm{0}} 15$, p. 415-428.

ENTMAN, R. M. (1993). "Framing: toward clarification of a fractured paradigm. En: Journal of Communication", $\mathrm{n}^{\circ} 43$ (4), p. 51-58.

FRANKLIN, B.; CARLSON, M. (2011). Journalism, Sources and Credibility. New Perspectives. London: Routledge.

GAMSON W. A.; MODIGLIANI, A. (1989). "Media discourse and public opinion on nuclear power: a constructionist approach". En: The American Journal of Sociology, $\mathrm{n}^{\circ} 95$ (1), p. 1-37.

GANS, H. J. (1980). Deciding What's News. A Study of CBS Evening News, NBC Nightly News, Newsweek and Time. New York: Vintage.

GARCÍA SANTAMARÍA, J. V. (2010). "Crisis del periodismo de fuentes. Las prácticas del periodismo en España en el accidente de Spanair". En: Revista Latina de Comunicación Social, no 65, p. 516-537.

GITLIN, T. (1980/2003). "The whole world is watching. Mass media in the making and unmaking of the new left. Berkeley (California): University of California Press.

GOFFMAN, E. (1974/2006). Frame analysis. Los marcos de la experiencia. Madrid: CIS. 
GROSSI, G. (2007). La opinión pública. Madrid: CIS.

IYENGAR, S. (1991). Is anyone responsible? How television frames political issues. Chicago: University of Chicago Press.

KAMHAWI, R.; WEAVER, D. (2003). "Mass communication research trends from 1980 to 1999". En: Journalism \& Mass Communication Quarterly, n 80 (1), p.7-27.

LEWIS, J.; WILLIAMS, A.; FRANKLIN, B. (2008). "A compromised Fourth Estate?". En: Journalism Studies, no 9 (1), p. 1-20.

LÓPEZ-RABADÁN, P. (2010). "Nuevas vías para el estudio del framing periodístico. La noción de estrategia de encuadre". En: Estudios sobre el Mensaje Periodístico, $\mathrm{n}^{\mathrm{o}} 16$, p. 235-258.

MANNING, P. (2001). News and news sources. A critical introduction. London: Sage.

MATTHES, J.; KOHRING, M. (2008). "The content analysis of media frames: toward improving reliability and validity". En: Journal of Communication, $\mathrm{n}^{\circ} 58$ (2), p. 258-279.

MAYORAL, J. (2005). "Fuentes de información y credibilidad periodística". En: Estudios sobre el Mensaje Periodístico, $\mathrm{n}^{\mathrm{o}}$ 11, p. 93-102.

McCOMBS, M. E. (2004). Setting the Agenda: The Mass Media and Public Opinion. Cambridge: Polity Press.

McCOMBS, M. E.; SHAW, D. L. (1972). "The agenda-setting function of mass media". En: Public Opinion Quarterly, no 36, pp. 176-187.

REESE, S. D.; GANDY, O. H. Jr.; GRANT, A. E. (eds.) (2001). Framing public life. Perspectives on media and our understanding of the social world. Mahwah (New Jersey): Lawrence Erlbaum.

SEMETKO, H. A.; VALKENBURG P. M. (2000). "Framing european politics: analysis of press and TV news". En: Journal of Communication, $\mathrm{n}^{\circ} 50$ (1), p.93-109.

TEWKSBURY, D.; SCHEUFELE, D. A, (eds.) (2007). "Special Issue on Framing, Agenda Setting, \& Priming: Agendas for Theory and Research". En: Journal of Communication, $\mathrm{n}^{\circ} 57$ (1), p. 9-173.

TUCHMAN, G. (1978). Making news: a study in the construction of reality. New York: The Free Press.

VICENTE-MARIÑO, M.; LÓPEZ-RABADÁN, P. (2009). "Resultados de la investigación sobre framing: sólido avance internacional y arranque de la especialidad en España". En: ZER. Revista de Estudios de Comunicación, no 14, 26, p.13-34.

WEAVER D. H. (2007). "Thoughts on agenda setting, framing and priming". En: Journal of Communication, $\mathrm{n}^{\circ} 57$ (1), p. 142-147.

\section{Notas}

1 Vinculado al proyecto de investigación CSO2010-16313 financiado por el Ministerio de Economía y Competitividad del Gobierno de España (Plan Nacional de I+D+i 2008-2011). 


\section{Los autores}

Pablo López-Rabadán. Profesor del Departamento de Ciencias de la Comunicación de la Universitat Jaume I de Castellón, donde imparte clases en el Grado en Periodismo y en el Máster Oficial en Nuevas Tecnologías y Procesos de Innovación en Comunicación. Ha sido investigador invitado en la Cardiff School of Journalism (JOMEC), en la University of Westminster y en Università degli Studi di Firenze. Sus líneas de investigación se centran en el periodismo político, y las nuevas tendencias en periodismo especializado y comunicación política. Desde 2005 ha participado en diversos proyectos $\mathrm{I}+\mathrm{D}$ y congresos científicos nacionales e internacionales. Ha publicado en revistas como Zer. Revista de Estudios en Comunicación, Estudios sobre el Mensaje Periodístico, Textual \& Visual Media, Revista Latina de Comunicación Social o American Behavioral Scientist.

Andreu Casero-Ripollés. Profesor titular y vicedecano-director del Grado en Periodismo de la Universitat Jaume I de Castelló (UJI). Co-director del Máster Oficial en Nuevas Tecnologías y Procesos de Innovación en Comunicación de la UJI. Ha sido profesor e investigador invitado en la Università degli Studi di Milano-Bicocca, en la Université Paris 8 y en la Universidade Estadual Paulista - UNESP. Es investigador principal de varios proyectos $\mathrm{I}+\mathrm{D}$. Sus líneas de investigación se centran en la comunicación política y la transformación del periodismo en el entorno digital. Ha publicado en revistas como Information, Communication \& Society, American Behavioral Scientist o Media International Australia. Recientemente, ha publicado, como editor, el libro Periodismo político en España: concepciones, tensiones y elecciones (SLCS, 2012). 